\title{
In situ TEM study of a new grain boundary deformation mechanism in olivine
}

PROF. PATRICK CORDIER ${ }^{1}$, IHTASHAM UL HAQ ${ }^{2}$, VAHID SAMAE $^{2}$, HOSNI IDRISSI ${ }^{3}$ AND DOMINIQUE SCHRYVERS $^{2}$

${ }^{1}$ Univ. Lille

${ }^{2}$ University of Antwerp

${ }^{3}$ Université Catholique de Louvain

Presenting Author: Patrick.Cordier@univ-lille.fr

Olivine is the dominant mineral phase in the Earth's upper mantle. There is a large body of compelling evidence that in the convective mantle, olivine deformation involves dislocation glide and climb. However, due to its low symmetry, this mineral does not possess enough slip systems to satisfy the Von Mises criterion for a general deformation. Several recent studies have focused on the possible contribution of grain boundaries (GBs) (sliding, migration) to the deformation of olivine aggregates, but so far, the mechanisms at play are not yet clarified. Recently, high resolution TEM microstructural investigation of olivine aggregates deformed at $\mathrm{ca} .1100{ }^{\circ} \mathrm{C}$ revealed a ductile behavior involving GB slinging involving $\mathrm{GB}$ amorphization.

Here we use in situ TEM nanomechanical testing on olivine aggregate samples (without amorphous layer at the GB) to gain information on the underlying deformation mechanisms. We use the PI-95 TEM Pico-indenter holder and the Push-to-Pull (PTP) device (Bruker. Inc) to perform quantitative in situ TEM tensile tests at room temperature. Bi- and tri-crystal olivine samples were prepared by focused ion beam (FIB). We show that the specimens deform exclusively by grain boundary sliding while observing evidence of stress-induced amorphization in the sliding GBs. The elementary mechanisms involved are discussed and compared to the literature. 